\title{
ORIGINAL ARTICLE \\ Predictors of pressure ulcer incidence following traumatic spinal cord injury: a secondary analysis of a prospective longitudinal study
}

\author{
D Brienza ${ }^{1,2,3}$, S Krishnan ${ }^{4}, \mathrm{P} \mathrm{Karg}^{1}$, G Sowa ${ }^{5,6}$ and AL Allegretti ${ }^{7}$
}

\begin{abstract}
Study design: Secondary analysis of data from a prospective cohort study.
Objectives: The objective of this study was to identify the medical and demographic factors associated with the development of pressure ulcers during acute-care hospitalization and inpatient rehabilitation following acute spinal cord injury.

Setting: The study was carried out at acute hospitalization, inpatient rehabilitation and outpatient rehabilitation sites at a university medical center in the United States.

Methods: Adults with acute traumatic spinal cord injury $(n=104)$ were recruited within $24-72 \mathrm{~h}$ of admission to the hospital. Pressure ulcer incidence was recorded.

Results: Thirty-nine participants out of 104 (37.5\%) developed at least one pressure ulcer during acute-care hospitalization and inpatient rehabilitation. Univariate logistic regression analyses revealed significant association of pressure ulcer incidence for those with pneumonia and mechanical ventilation $(P=0.01)$ and higher injury severity (ASIA A) $(P=0.01)$. Multiple logistic regression showed that the odds of formation of a first pressure ulcer in participants with ASIA A was 4.5 times greater than that for participants with ASIA B, CI (1-20.65), $P=0.05$, and 4.6 times greater than that for participants with ASIA C, CI (1.3-16.63), $P=0.01$.

Conclusion: Among individuals with acute traumatic $\mathrm{SCl}$, those with high-injury severity were at an increased risk to develop pressure ulcers. Pneumonia was noted to be associated with the formation of pressure ulcers.
\end{abstract}

Spinal Cord (2018) 56, 28-34; doi:10.1038/sc.2017.96; published online 12 September 2017

\section{INTRODUCTION}

Spinal cord injury (SCI) is a neurologic disorder with profound physical, psychological and socioeconomic impacts. ${ }^{1}$ Increase in age and severity of injury are associated with long-term complications after traumatic SCI. ${ }^{2}$ The second leading cause of death in individuals with SCI is septicemia $(88.6 \%)$; usually associated with urinary tract infections (UTIs), pneumonia or presence of pressure ulcers. ${ }^{3}$ Pressure ulcers are the second most frequent secondary complication in individuals with SCI from the time of acute hospitalization through community reintegration. ${ }^{4}$ Pressure ulcers affect quality of life, length of stay during hospitalization and increase mortality and morbidity. ${ }^{5-8}$

Many risk factors have been associated with the formation of pressure ulcers. Medical complications, such as cardiac or renal disease, have been associated with increased risk. ${ }^{9}$ Individuals likely to develop pressure ulcers are: those who have SCI of traumatic origin; ${ }^{11}$ men ${ }^{12}$ with a history of smoking, alcohol or drug use; ${ }^{13}$ those with medical comorbidities such as diabetes mellitus, ${ }^{14,15}$ decreased oxygenation or hypotension; ${ }^{16}$ those with infections such as pneumonia, urinary tract infections, osteomyelitis and other bacterial infections; ${ }^{10,17}$ those on mechanical ventilators; ${ }^{18}$ and those who use steroids. ${ }^{16}$ In the acute-care and intensive-care healthcare environments, moisture and urinary and fecal incontinence, hypo/hyperthermia and shear, have been reported as extrinsic risk factors for the formation of pressure ulcers. ${ }^{13,15,18-22}$ Decreased nutrition or low serum albumin levels, decreased mobility and sensation, and impaired cognitive function are intrinsic factors shown to contribute to risk. ${ }^{13,14}$

The environment plays an important role in the development of pressure ulcers. Most studies focus on the risk factors associated with the development of pressure ulcers during community care for individuals with SCI, ${ }^{5,9,12,23-27}$ however less address the risk factors in this population during hospitalization or acute care. ${ }^{15}$ Hence, identifying the factors for development of pressure ulcers for individuals with SCI in high-risk settings (for example, during hospitalization) is essential. Studies on risk factors associated with pressure ulcers most often used self-report or retrospective chart review methods. ${ }^{9,12}$ These methods may result in under-reporting of the data due to recall bias or misclassification of the data. Although numerous risk factors are associated with the occurrence of pressure ulcers, those factors related to increased incidence for newly injured

${ }^{1}$ Department of Rehabilitation Science and Technology, School of Health and Rehabilitation Science, University of Pittsburgh, Pittsburgh, PA, USA; ${ }^{2}$ McGowan Institute for Regenerative Medicine, University of Pittsburgh, Pittsburgh, PA, USA; ${ }^{3}$ Department of Bioengineering, University of Pittsburgh, Pittsburgh, PA, USA; ${ }^{4}$ Department of Occupational Therapy, University of Texas Medical Branch, Galveston, TX, USA; ${ }^{5}$ Department of Physical Medicine and Rehabilitation, University of Pittsburgh, School of Medicine, Pittsburgh, PA, USA; ${ }^{6}$ Ferguson Laboratory for Orthopaedic Research, Department of Orthopaedic Surgery, University of Pittsburgh, School of Medicine, Pittsburgh, PA, USA and ${ }^{7}$ Department of Occupational Therapy, UT Health Science Center, San Antonio, TX, USA

Correspondence: Dr D Brienza, Department of Rehabilitation Science and Technology, School of Health and Rehabilitation Science, University of Pittsburgh, 6425 Penn Ave, Suite 401, Pittsburgh, PA 15206, USA.

E-mail: dbrienza@pitt.edu

Received 17 March 2017; revised 11 July 2017; accepted 12 July 2017; published online 12 September 2017 
individuals with SCI during hospitalization have not been adequately determined.

The purpose of this study was to identify the medical and demographic factors associated with the development of pressure ulcers during acute-care hospitalization and inpatient rehabilitation so that the information may help prevent pressure ulcers, reduce the risk of re-hospitalization and improve quality of life. We hypothesized medical factors such as presence of pneumonia, UTI, use of steroids and demographic factors such as gender, age and severity of injury measured by American Spinal Injury Association (ASIA) would be associated with increased risk for the formation of pressure ulcers in newly injured individuals with traumatic SCI during acute-care hospitalization and inpatient rehabilitation.

\section{METHODS}

\section{Research design}

This study was conducted at the Rehabilitation Engineering Research Center on Spinal Cord Injury (RERC on SCI) at the University of Pittsburgh and UPMC Medical Center. UPMC Medical Center provides acute care with specialized neurology and neurosurgery services for individuals with spinal cord injury. Inpatient rehabilitation care was provided in a unit dedicated to spinal cord injury rehabilitation, where patient care is led by physicians from the Department of Physical Medicine and Rehabilitation. The study is a secondary analysis of data collected on newly injured individuals with traumatic SCI during acute and inpatient rehabilitation. The RERC on SCI had the broad directive from its sponsor, the National Institute on Disability and Rehabilitation Research, to serve individuals with SCI through research and development of new technologies to improve treatment, and thus help with reintegration into society. The Center enrolled acute patients with SCI and collected blood, urine, demographic information and medical information during their acute and inpatient rehabilitation stay at UPMC and post discharge. The primary aim was to obtain information related to the incidence of pressure ulcers, the occurrence of urinary tract infections, data associated with depression and pain status, with the goal to develop a model of inflammation and healing in pressure ulcer development following SCI. ${ }^{28}$

The RERC protocol received approval from the University of Pittsburgh Institutional Review Board. After obtaining informed consent, clinical data, plasma and urine samples were collected three times per week when the subject was in acute care, weekly in inpatient rehabilitation and annually after discharge to outpatient care for the duration of the study, up to 5 years (2008-2012). This secondary analyses explores and examines the relationships among the medical and demographic variables and pressure ulcer outcomes. It focuses on determining predictors for incidence of first pressure ulcer. Factors included in the statistical prediction models were limited to those recorded for the primary aim of the original study.

\section{Inclusion and exclusion criteria}

In collaboration with University of Pittsburgh Model System on SCI, most participants were recruited within $24-72 \mathrm{~h}$ of admission to the hospital. The potential participants were eligible for the study if they met the following inclusion criteria-received acute medical and surgical treatment or were admitted to inpatient rehabilitation at UPMC hospitals, were 18 years and older and presented with acute traumatic SCI. The potential participants were excluded if they had the following comorbidities-pre-existing diseases (such as autoimmune or demyelinating diseases) that affected the inflammatory response to SCI, previous SCI or other neurological diseases that affected the motor and sensory function. Potential participants with diabetes were initially excluded from the study, but later included after the first year of data collection in an effort to increase enrollment.

\section{Data collection}

Pressure ulcer risk variables included age, gender, level of injury, other injuries associated with trauma, ASIA Impairment Scale (AIS), smoking, alcohol use and comorbidities. ${ }^{5,29}$ The AIS was used to grade severity of motor and sensory impairment. ${ }^{30}$ Injury severity score, was used to record the combined severity of other traumatic injuries. ${ }^{31}$ The AIS and injury severity score were noted from the UPMC electronic health records by the research staff. If a participant developed a pressure ulcer, the research nurses recorded the severity, size, shape and progression of the wound healing. Severity was determined using National Pressure Ulcer Advisory Panel staging system. ${ }^{32}$ The medications and treatment protocols such as steroids, NSAIDs, pain, anti-inflammatory and any other mediation these participants were on were noted from their electronic health records.

\section{Procedure}

The primary outcome was occurrence of the first pressure ulcer. Categories of pressure ulcers considered for analysis included stage I-IV, suspected deep tissue injury and unstageable. The list of possible predictors was reduced to the following for analysis, based upon the expert opinion of a group of clinicians and review of the literature: age, AIS score, gender, use of steroids, urinary tract infection diagnosis, pneumonia diagnosis, use of mechanical ventilation and diabetes diagnosis. Use of mechanical ventilation was defined as using a ventilator, having tracheostomy or endotracheal tube, or using continuous positive airway pressure.

\section{Analysis}

All data were analyzed using Statistical Package for Social Sciences (SPSS, IBM, Armonk, NY, USA), version 20.0. Descriptive statistics using means, frequencies and standard error of mean were computed. A univariate logistic regression analysis was conducted to assess individual risk factors' ability to predict the probability of the outcome of first pressure ulcer (yes/no) during acute care or inpatient rehab. Diabetes diagnosis was a special case in the analysis because it was an exclusion criterion for the first year of the study. During the time when subjects with diabetes were excluded, 17 subjects were enrolled. We performed the univariate analysis on diabetes with and without including these 17 subjects. A multiple binary logistic regression (simultaneous method) was conducted with the independent variables selected from univariate analysis and by reviewing the literature that previously identified these risk factors. The outcome for the model was occurrence of first pressure ulcer (yes/no). As a secondary analysis, sample size was not determined before the data collection. Peduzzi et al..$^{33}$ determined that 10 cases per predictor variable would produce a valid model fit to justify the sample size. The assumptions of logistic regression, such as multicollinearity, linearity and outliers, were checked to minimize the number of predictors and include the best set of variables to maximize the strength of prediction. The Hosmer-Lemeshow goodness of fit was computed to evaluate the goodness of fit by comparing the observed model to the predicted logistic regression model. The receiver operating characteristic (ROC) curve was plotted and the area under the ROC curve (AUC) was calculated for the multiple logistic regression model to quantify the power and evaluate the accuracy of the multiple logistic regression model. An AUC varies from 0.5 (represents discriminating power not better than chance) to 1.0 (represents a perfect discriminating power). ${ }^{34}$ The significance level was set at $\alpha=0.05 a$ priori.

\section{RESULTS}

\section{Participants}

One hundred and four individuals were enrolled in the RERC on SCI study. The demographics, characteristics and medical comorbidities of the sample population are shown in Table 1. The majority of participants' spinal cord injuries were due to falls (38\%) and motor vehicle accidents $(32 \%)$. The participants were followed for variable time periods through their stays in acute care and inpatient rehabilitation. Follow up terminated in acute care for 29 participants and in inpatient rehabilitation for 28 participants. Four participants began follow-up in inpatient rehabilitation. None of these four participants entered the study with an existing pressure ulcer. The mean follow-up period was 35.9 days with an s.d. of 28.2 days. Eighty-one percent of the participants in the population were Caucasians and $80 \%$ were male. Almost $93 \%$ of participants in acute care and $83 \%$ of 
Table 1 Demographics, characteristics and medical comorbidities for all particpants in the study and subgroups made up of those that developed a pressure ulcer in acute care or in inpatient rehabilitation and those that did not developed a pressure ulcer in acute care or in inpatient rehabilitation

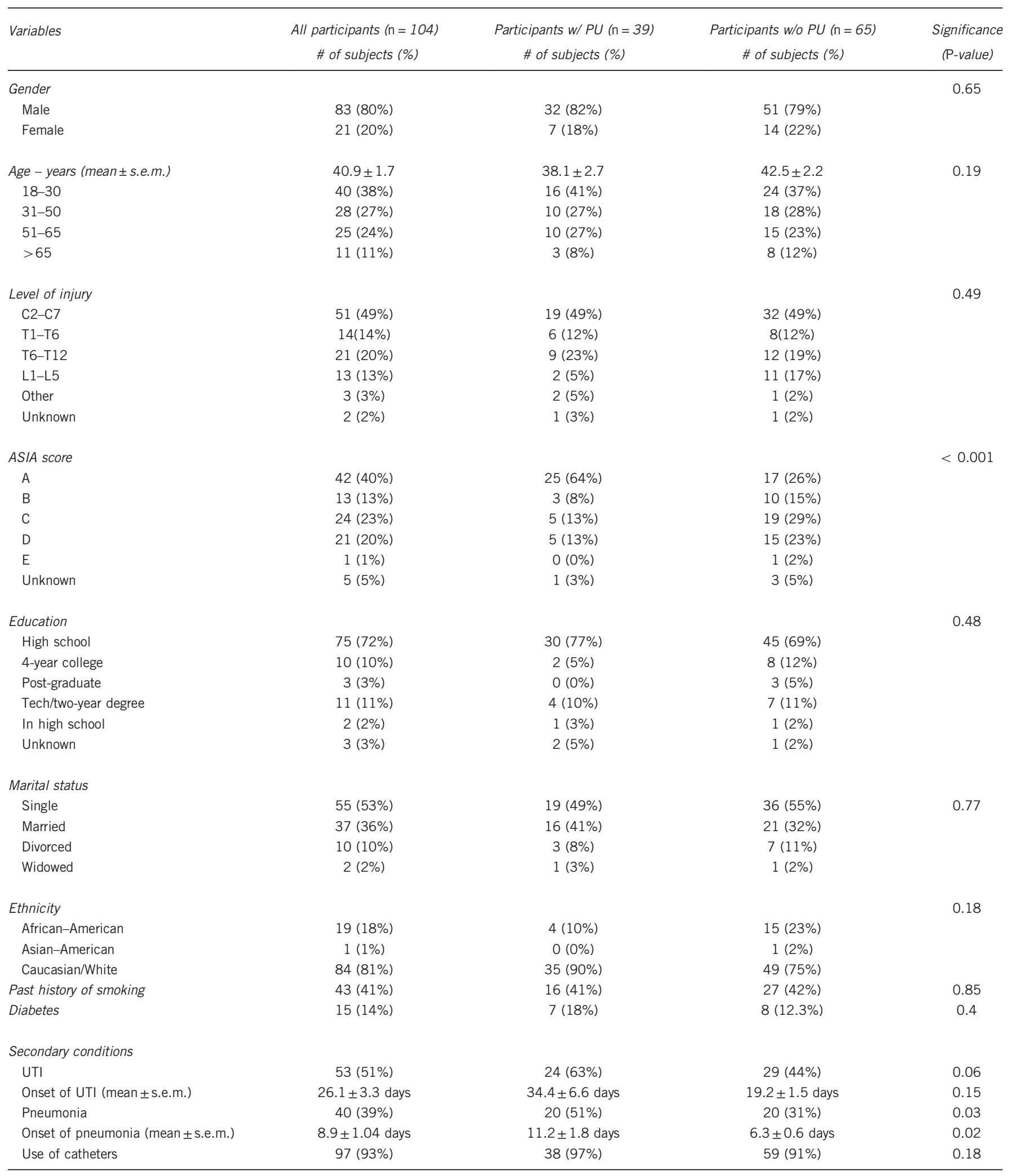

Abbreviation: ASIA, American Spinal Injury Association. 
participants in inpatient rehabilitation used catheters for their bladder management.

\section{Incidence of pressure ulcers}

Thirty-nine participants out of 104 (37.5\%) developed at least one ulcer during acute care or inpatient rehabilitation. The incidence of a first pressure ulcer was $27 \%(n=28)$ during follow-up in acute care and $12 \%(n=12)$ during follow-up inpatient rehabilitation. The severity of the first occurring pressure ulcer for most of the participants in this sample was Stage II. The overall distribution of severity was Stage I $(n=3,8 \%)$, Stage II $(n=25,64 \%)$, Stage III $(n=4,10 \%)$, Stage IV $(n=3,8 \%)$, sDTI $(n=2,5 \%)$ and unstageable $(n=2,5 \%)$. The mean number of days from the time of SCI until onset of the first pressure ulcer was $20.2 \pm 11.5$ days for participants in acute care hospitalization and inpatient rehabilitation.

The demographics, characteristics and medical comorbidities of all participants $(n=104)$ are shown in Table 1 , broken down by those participants who did and did not develop pressure ulcers during acute care or inpatient rehabilitation. Significant differences in distribution of ASIA scores, occurrence of UTI and occurrence and onset of pneumonia were found between those who did and did not develop a pressure ulcer.

\section{Predictors of occurrence of first pressure ulcer during acute care and inpatient rehabilitation}

A univariate logistic regression was performed for age, gender, ASIA classification, pneumonia, UTI, steroids use and diabetes (Table 2). The analysis was significant for participants with pneumonia $(P=0.01)$, mechanical ventilation $(P=0.01)$ and for injury severity (ASIA) $(P=0.01)$. The average number of days from the time of injury to development of pneumonia in participants with pressure ulcers in this study was 11 days. The ROC curve was calculated for all the individual predictors except diabetes and the AUC was computed (Table 3). The AUC for participants who had pneumonia was 0.63 and significant $(P=0.04)$.

An additional univariate logistic regression analysis was performed on the participants enrolled after the elimination of diabetes diagnosis

Table 2 Univariate logistic regression of clinical and demographic factors $(n=104)$

\begin{tabular}{|c|c|c|c|}
\hline Variables & $\begin{array}{l}\text { Individuals with at least } 1 \\
\text { PU in acute care through } \\
\text { inpatient rehabilitation } \\
n=39\end{array}$ & $\begin{array}{l}\text { Individuals with no PU } \\
\text { in acute care through } \\
\text { inpatient rehabilitation } \\
\qquad \mathrm{n}=65\end{array}$ & $\begin{array}{c}\text { Significance } \\
\text { (P) }\end{array}$ \\
\hline Age (mean) & 38.12 years & 42.50 years & 0.22 \\
\hline Gender (N) & $\begin{array}{c}M=32 \\
F=7\end{array}$ & $\begin{array}{l}M=51 \\
F=14\end{array}$ & 0.79 \\
\hline ASIA (N) & $\begin{array}{l}A=25 \\
B=3 \\
C=5 \\
D=5\end{array}$ & $\begin{array}{l}A=17 \\
B=10 \\
C=19 \\
D=15\end{array}$ & $0.01^{*}$ \\
\hline Pneumonia (N) & $Y=21$ & $Y=19$ & $0.01 *$ \\
\hline UTI (N) & $Y=24$ & $Y=29$ & 0.09 \\
\hline Steroids (N) & $Y=13$ & $Y=20$ & 0.78 \\
\hline Diabetes (N) & $Y=7$ & $Y=8$ & 0.43 \\
\hline $\begin{array}{l}\text { Mechanical } \\
\text { ventilation (N) }\end{array}$ & $Y=25$ & $Y=25$ & $0.01 *$ \\
\hline
\end{tabular}

Abbreviation: ASIA, American Spinal Injury Association.

${ }^{*} P<0.05$ as an exclusion criterion. This analysis included 87 participants, 15 with diabetes diagnosis. Diabetes diagnosis was not significant $(P=0.39)$. This subgroup analysis and result suggests that the mid-study change to the diabetes inclusion criteria did not significantly skew the univariate analysis of the effect of diabetes for the entire study sample.

A multiple binary logistic regression (simultaneous method) was performed with pressure ulcer as the outcome and six predictors: age, ASIA classification, gender, steroid use, pneumonia and urinary tract infection. The pressure ulcer outcome was dichotomized into two levels (present (1) or not present (0)). The two levels in UTI, steroids and pneumonia were yes (1) and no (0). The two levels in gender were male and female, and the four levels in severity of injury were (ASIA A (1), ASIA B (2), ASIA C (3) and ASIA D (4)). Table 4 shows the results of the multiple logistic regression. All assumptions were met. A significant prediction of pressure ulcer outcome by the medical and demographic factors included in the multiple model, $\chi 2(9)=19.08$, $P=0.02$, was found. The odds of formation of a first pressure ulcer in participants with ASIA A was 4.5 times greater than for participants with ASIA B, CI (1-20.65), $P=0.05$, and the odds of formation of a pressure ulcer in participants with ASIA A was 4.6 times greater than for participants with ASIA C, CI (1.3-16.63), $P=0.01$. No significant prediction of pressure ulcer occurrence by medical factors such as pneumonia $(P=0.7)$, urinary tract infection $(P=0.09)$, use of steroids $(P=0.32)$, mechanical ventilation $(P=0.25)$ and demographic factors such as age $(P=0.76)$ and gender $(P=0.6)$ was found. No differences were found between the observed and predicted group membership, Hosmer-Lemeshow $\chi^{2}(8)=9.89, P=0.27$, indicating a good overall fit of the logistic regression model and no misspecification of the predictors. The area under the ROC curve (Figure 1) was 0.74 with $95 \%$ CI $(0.64,0.84),(P \leqslant 0.0001)$, indicating that the variables in the multiple logistic regression model significantly classified formation of pressure ulcers in the study population.

\section{DISCUSSION}

The overall pressure ulcer incidence rate of at least one pressure ulcer in the study population during acute hospitalization and inpatient rehabilitation was $38 \%$. The observed rate is lower when compared with the rates that were reported by an earlier study indicating that $\sim 47 \%$ of participants with SCI develop at least one pressure ulcer during the period of acute-care hospitalization and rehabilitation. ${ }^{35}$ However, the rate is consistent with the 2011 Model Systems on Spinal Cord Injury, which reported that $34 \%$ of individuals develop at least one pressure ulcer during acute hospitalization and inpatient rehabilitation. ${ }^{3}$ Most of the pressure ulcers in earlier studies occurred in acute care as compared with that in rehabilitation units, ${ }^{36,37}$ these previous findings are comparable to the present study. The incidence of pressure ulcers in this study was $27 \%$ during acute care and $12 \%$ during inpatient rehabilitation.

In this study, participants with severity classification of ASIA A developed pressure ulcers at higher rates than those classified as ASIA B or C, similar to previous studies. ${ }^{38}$ Scheel-Sailer et al..$^{39}$ performed a prospective cohort study in individuals with SCI and found similar completeness of injury a significant risk, where the odds of forming a pressure ulcer with ASIA A were much higher than ASIA C or D. A complete motor and sensory lesion (ASIA A) severely limits mobility and independent performance of other activities of daily living. ${ }^{27,38,40,41}$ In addition, individuals with an ASIA A classification of injury are typically on anesthesia and mechanical ventilation. These conditions decrease their awareness of pressure and shear forces on their bony prominences. Additional factors predisposing individuals with higher severity to pressure ulcers are urinary and/or fecal 
Table 3 AUC for all variables

\begin{tabular}{|c|c|c|c|c|c|}
\hline \multirow[t]{2}{*}{ Variables } & \multirow[t]{2}{*}{ Area } & \multirow[t]{2}{*}{ s.e. } & \multirow[t]{2}{*}{$\begin{array}{c}\text { Significance } \\
\text { (P) }\end{array}$} & \multicolumn{2}{|c|}{$\begin{array}{c}\text { Asymptotic 95\% confi- } \\
\text { dence interval }\end{array}$} \\
\hline & & & & $\begin{array}{l}\text { Upper } \\
\text { bound }\end{array}$ & $\begin{array}{l}\text { Lower } \\
\text { bound }\end{array}$ \\
\hline ASIA A-ASIA B & 00.459 & 0.059 & 0.49 & 0.344 & 0.574 \\
\hline ASIA A-ASIA C & 00.413 & 0.057 & 0.14 & 0.300 & 0.525 \\
\hline ASIA A-ASIA D & 00.445 & 0.058 & 0.36 & 0.330 & 0.559 \\
\hline Age & 00.423 & 0.059 & 0.20 & 0.306 & 0.540 \\
\hline Gender & 00.474 & 0.059 & 0.67 & 0.358 & 0.590 \\
\hline Pneumonia & 00.626 & 0.059 & $0.04 *$ & 0.511 & 0.741 \\
\hline UTI & 00.569 & 0.059 & 0.25 & 0.453 & 0.685 \\
\hline Mechanical ventilation & 00.628 & 0.057 & $0.03^{*}$ & 0.517 & 0.739 \\
\hline Steroids & 00.505 & 0.060 & 0.94 & 0.387 & 0.622 \\
\hline
\end{tabular}

Abbreviation: ASIA, American Spinal Injury Association.

${ }^{*} P<0.05$.

Table 4 Multiple logistic regression of clinical and demographic factors

\begin{tabular}{|c|c|c|c|c|c|}
\hline Independent variables & B & s.e. & Wald & Significance (P) & $\operatorname{Exp}(B) C l$ \\
\hline Age & -0.00 & 0.14 & 0.08 & 0.76 & $\begin{array}{c}0.99 \\
0.96-1.02\end{array}$ \\
\hline Gender & 0.3 & 0.57 & 0.27 & 0.6 & $\begin{array}{c}1.35 \\
0.43-4.16\end{array}$ \\
\hline ASIA A-ASIA B & 1.51 & 0.77 & 3.85 & $0.05^{*}$ & $\begin{array}{c}4.55 \\
1-20.65\end{array}$ \\
\hline ASIA A-ASIA C & 1.53 & 0.64 & 5.61 & $0.01^{*}$ & $\begin{array}{c}4.66 \\
1.3-16.63\end{array}$ \\
\hline ASIA A-ASIA D & 0.74 & 0.73 & 1.02 & 0.31 & $\begin{array}{c}2.09 \\
0.49-8.80\end{array}$ \\
\hline Pneumonia & -0.19 & 0.57 & 0.11 & 0.7 & $\begin{array}{c}0.82 \\
0.26-2.55\end{array}$ \\
\hline Urinary tract infection & -0.79 & 0.47 & 2.83 & 0.09 & $\begin{array}{c}0.45 \\
0.17-1.14\end{array}$ \\
\hline Steroids & -0.48 & 0.49 & 0.96 & 0.32 & $\begin{array}{c}0.61 \\
0.23-1.63\end{array}$ \\
\hline Mechanical ventilation & -0.66 & 0.58 & 1.3 & 0.25 & $\begin{array}{c}0.51 \\
0.16-1.60\end{array}$ \\
\hline Constant & -2.6 & 1.77 & 2.16 & 0.14 & 0.07 \\
\hline
\end{tabular}

Abbreviation: ASIA, American Spinal Injury Association.

The dependent variable in this analysis is formation of pressure ulcer in acute care or inpatient rehabilitation, so that $0=$ did not have pressure ulcer and $1=$ had a formation of pressure ulcer. ${ }^{*} P<0.05$.

Model $\chi^{2}=19.08, P<0.05$

Pseudo $R^{2}=0.22$.

$n=104$.

incontinence resulting in increased moisture on and maceration of the skin. $^{42}$

The univariate analysis revealed pneumonia as another factor that predicted pressure ulcer outcome. On average, participants in this study were diagnosed with pneumonia within 11 days following injury. The area under the ROC determined that participants with pneumonia have greater occurrence of pressure ulcers than participants with no pneumonia, indicating a relationship between pulmonary disease and formation of pressure ulcers. Pneumonia was the only medical comorbidity that predicted occurrence of pressure ulcers in the univariate analysis, but was not significant in presence of other variables that were included in the multiple logistic regression model. Increased severity of injury and complete loss of motor control predisposes individuals with SCI to pneumonia due to ventilatory incompliance. Hence, individuals with higher level and complete SCI are dependent on mechanical ventilation. The univariate analysis also revealed mechanical ventilation to be associated with formation of pressure ulcers. Individuals dependent on mechanical ventilators' lack of movement may predispose them to develop pressure ulcers. Correlation between pneumonia and the formation of pressure ulcers has been indicated in previous studies. ${ }^{13,24,27,43}$ A previous study also revealed association between pneumonia, mechanical ventilation and pressure ulcer. ${ }^{44}$ The pathogenesis between the formation of pressure ulcers and presence of pneumonia may be related to an inflammatory response. ${ }^{45}$

Demographics, such as age and gender, were not significant predictors of pressure ulcer formation in this study. Although some other studies have identified age ${ }^{2}$ and gender ${ }^{11}$ as being associated with pressure ulcer formation, a recent study by Wilczweski and colleagues, determined demographics not to be a significant predictor. ${ }^{15}$

Fifty-one percent of all participants developed a UTI within an average of 26 days from injury, whereas $63 \%$ of participants with pressure ulcers developed UTIs within an average of 34 days from injury. Diagnosis of UTI was not a significant predictor of pressure ulcer development. Other studies have indicated UTIs to be associated with the risk of development of pressure ulcers in acute care hospitals. ${ }^{16}$ Most of the individuals $(80 \%)$ in hospitals are predisposed to UTIs because of the use of catheters for bladder management and improper maintenance and insertion of catheters. ${ }^{46,47}$ Almost all the participants (93\% during acute care and $85 \%$ during inpatient rehabilitation) used catheters for bladder management. These large percentages may have contributed to the reason why UTI was not found to be a predictor of PU in the multiple regression model.

Diabetes is also known to be associated with the formation of pressure ulcers. ${ }^{16,48,49}$ Diabetes was not found to be significant predictor in this study's sample in the univariate analysis. The use of steroids was a not significant predictor of pressure ulcer development in either the univariate or multiple logistic regression analyses. The evidence regarding the use of steroids and formation of pressure ulcers is inconclusive. In a previous study, the use of steroids was significant in the univariate analysis and not significant in the multiple logistic regression model. ${ }^{15}$

\section{Study limitations}

A potential limitation to this study is the small sample size: only 104 participants were recruited. The general 'rule of thumb' for a good model fit, to have 10 cases per predictor variable, was considered. ${ }^{33}$ Hence, only seven variables were included in the final multiple logistic regression model. Also, since the research design for this study was a secondary analysis, there were inherent disadvantages. Other possible variables, such as Braden scale score, depression score and pain scale at admission, could not be studied for association with the formation of pressure ulcers. The timing of diagnosis of certain comorbidities such as cardiovascular heart disease, nutrition, sepsis, hypotension and interventions such as dietary protein and use of support surfaces were not collected or analyzed. Twenty-nine participants' follow up terminated in acute care, which may have resulted in an underestimation of pressure ulcers if any of these participants went on to develop a first pressure ulcer in inpatient rehabilitation. Only a limited number of factors were identified in this study for the occurrence of pressure ulcers. Hence, it is essential to document additional medical factors from the electronic health records or medical records, if the 


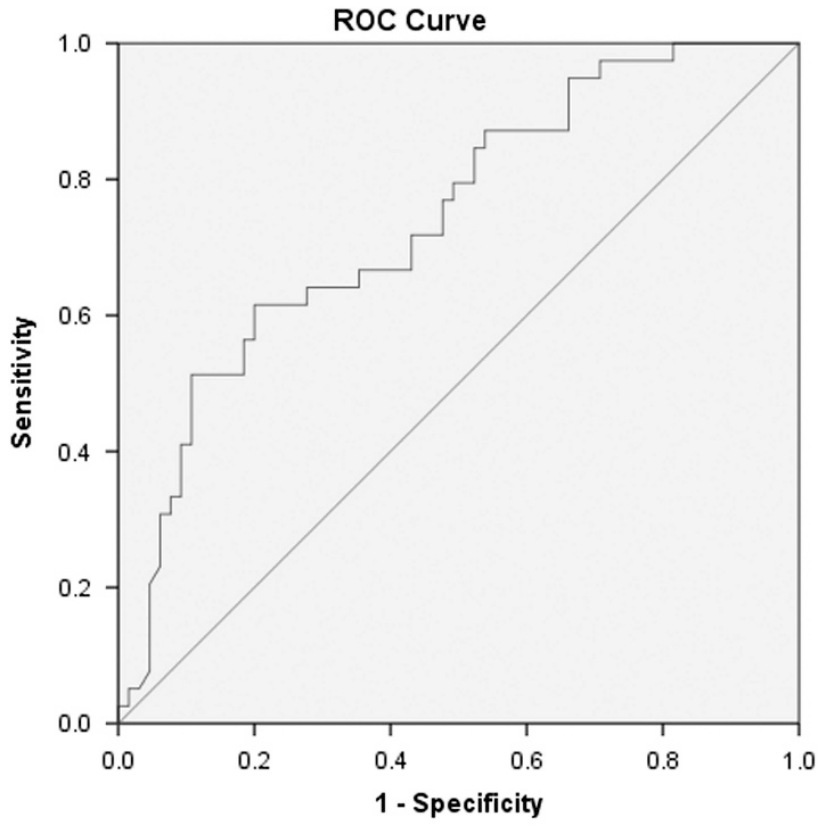

Diagonal segments are produced by ties.

Figure 1 ROC curve for multiple binary logistic regression (simultaneous method). A full colour version of this figure is available at the Spinal Cord journal online.

research staff is not able to obtain information on risk factors or comorbidities at the time of data collection. In addition, it may be interesting to explore the association between medical and rehabilitation interventions that these individuals were using and adherence to a prescribed preventive care plan and pressure ulcer incidence. Neither of these factors were considered as part of this study because we focused on the medical conditions for which we had data secondary to the parent study's methodology. Future research should include these other important factors.

\section{CONCLUSIONS}

Many risk factors have been associated with the formation of pressure ulcers. The participants with SCI are at a higher risk to develop pressure ulcers during the time of hospitalization. The study confirmed that individuals with high-injury severity are at increased risk to develop pressure ulcers. This study also suggests that the medical comorbidity pneumonia is associated with the formation of pressure ulcers.

\section{DATA ARCHIVING}

There were no data to deposit.

\section{CONFLICT OF INTEREST}

The authors declare no conflict of interest.

\section{ACKNOWLEDGEMENTS}

This work was funded by the National Institute on Disability and Rehabilitation Research (NIDRR), Rehabilitation Engineering Research Center (RERC) on Spinal Cord Injury, Grant \#H133E070024. Additional support was received from the NIDILRR Interdisciplinary Rehabilitation Research Training Program (Postdoctoral) Grant \#90AR5009.
1 Dumont RJ, Okonkwo DO, Verma S, Hurlbert RJ, Boulos PT, Ellegala DB et al. Acute spinal cord injury, part I: pathophysiologic mechanisms. Clin Neuropharmacol 2001; 24: 254-264.

2 Hitzig SL, Tonack M, Campbell KA, McGillivray CF, Boschen KA, Richards $\mathrm{K}$ et al. Secondary health complications in an aging Canadian spinal cord injury sample. Am J Phys Med Rehabil 2008; 87: 545-555.

3 NSCISC. Annual report for the model spinal cord injury care systems. N.S.C.I.S. Center: Birmingham, AL, 2011.

4 National Spinal Cord Injury Statistical Center. Annual report for the model spinal cord injury care systems. N.S.C.I.S. Center: Birmingham, AL, 2006.

5 Garber SL, Rintala DH, Hart KA, Fuhrer MJ. Pressure ulcer risk in spinal cord injury: predictors of ulcer status over 3 years. Arch Phys Med Rehabil 2000; 81: 465-471.

6 Cardenas DD, Hoffman JM, Kirshblum S, McKinley W. Etiology and incidence of rehospitalization after traumatic spinal cord injury: a multicenter analysis. Arch Phys Med Rehab 2004; 85: 1757.

7 Krause JS, Saunders LL. Health, secondary conditions, and life expectancy after spinal cord injury. Arch Phys Med Rehabil 2011; 92: 1770-1775.

8 Savic G, Short D, Weitzenkamp D, Charlifue S, Gardner B. Hospital readmissions in people with chronic spinal cord injury. Spinal Cord 2000; 38: 371.

9 Salzberg C, Byrne DW, Cayten GC, Kabir R, van Niewerburgh P, Viehbeck M et al. Predicting and preventing pressure ulcers in adults with paralysis. Adv Skin Wound Care 1998; 11: 237-246.

10 McKinley W, Tewksbury M, Godbout C. Comparison of medical complications following nontraumatic and traumatic spinal cord injury. J Spinal Cord Med 2002; 25: 88-93.

11 Chen D, Apple DF Jr, Hudson LM, Bode R. Medical complications during acute rehabilitation following spinal cord injury-current experience of the Model Systems. Arch Phys Med Rehabil 1999; 80: 1397.

12 Krause JS, Vines CL, Farley TL, Sniezek J, Coker J. An exploratory study of pressure ulcers after spinal cord injury: Relationship to protective behaviors and risk factors. Arch Phys Med Rehabil 2001; 82: 107-113.

13 Salzberg CA, Byrne DW, Cayten CG, van Niewerburgh P, Murphy JG, Viehbeck M. A new pressure ulcer risk assessment scale for individuals with spinal cord injury1. Am J Phys Med Rehab 1996; 75: 96-104.

14 Çakmak SK, Gül Ü, Özer S, Yigit Z, Gönü M. Risk factors for pressure ulcers. Adv Skin Wound Care 2009; 22: 412-415.

15 Wilczweski P, Grimm D, Gianakis A, Gill B, Sarver W, McNett M. Risk factors associated with pressure ulcer development in critically ill traumatic spinal cord injury patients. J Trauma Nurs 2012; 19: 5-10.

16 Fogerty MD, Abumrad NN, Nanney L, Arbogast PG, Poulose B, Barbul A. Risk factors for pressure ulcers in acute care hospitals. Wound Repair Regen 2008; 16: 11-18.

17 Manzano F, Navarro MJ, Roldán D, Moral MA, Leyva I, Guerrero C et al. Pressure ulcer incidence and risk factors in ventilated intensive care patients. J Crit Care 2010; 25: 469-476.

18 Banks M, Graves N, Bauer J, Ash S. Cost effectiveness of nutrition support in the prevention of pressure ulcer in hospitals. Eur J Clin Nutr 2012; 67: 42-46.

19 Watts D, Abrahams E, MacMillan C, Sanat J, Silver R, Van Gorder S et al. Insult after injury: pressure ulcers in trauma patients. Orthop Nurs 1998; 17: 84-91.

20 Reddy M, Gill SS, Rochon PA. Preventing pressure ulcers: a systematic review. JAMA 2006; 296: 974-984.

21 Peerless JR, Davies A, Klein D, Yu D. Skin complications in the intensive care unit. Clin Chest Med 1999; 20: 453-467.

22 Stover SL, DeLisa JA, Whiteneck GG. Spinal Cord Injury: Clinical Outcomes From The Model Systems. Aspen Publishers, New York, NY, USA, 1995.

23 Rodriguez G, Garber S. Prospective study of pressure ulcer risk in spinal cord injury patients. Spinal Cord 1994; 32: 150-158.

24 Gelis A, Dupeyron A, Legros P, Benaim C, Pelissier J, Fattal C. Pressure ulcer risk factors in persons with spinal cord injury part 2: the chronic stage. Spinal Cord 2009; 47: 651-661.

25 Garber SL, Rintala DH. Pressure ulcers in veterans with spinal cord injury: a retrospective study. J Rehabil Res Dev 2003; 40: 433-442.

26 Correa G, Fuentes M, Gonzalez X, Cumsille F, Pineros J, Finkelstein J. Predictive factors for pressure ulcers in the ambulatory stage of spinal cord injury patients. Spinal Cord 2006; 44: 734-739.

27 Chen $Y$, DeVivo MJ, Jackson AB. Pressure ulcer prevalence in people with spinal cord injury: age-period-duration effects. Arch Phys Med Rehabil 2005; 86: 1208-1213.

28 Ziraldo C, Solovyev A, Allegretti A, Krishnan S, Henzel MK, Sowa GA et al. A computational, tissue-realistic model of pressure ulcer formation in individuals with spinal cord injury. PLoS Comput Biol 2015; 11: e1004309.

29 Byrne D, Salzberg C. Major risk factors for pressure ulcers in the spinal cord disabled: a literature review. Spinal Cord 1996; 34: 255-263.

30 Marino RJ, Barros T, Biering-Sorensen F, Burns SP, Donovan WH, Graves DE et al. International standards for neurological classification of spinal cord injury. J Spinal Cord Med 2003; 26: S50.

31 Baker SP, o'Neill B, Haddon W Jr, Long WB. The injury severity score: a method for describing patients with multiple injuries and evaluating emergency care. J Trauma 1974; 14: 187-196.

32 NPUAP/EPUAP/PPPIA. National Pressure Ulcer Advisory Panel/European Pressure Ulcer Advisory Panel/Pan Pacific Pressure Injury Alliance: Prevention and Treatment of Pressure Ulcers: Clinical Practice Guideline. Haesler E (ed.). NPUAP/EPUAP/PPPIA. Perth, Australia, 1-308, 2014. Available at http://internationalguideline.com. 
33 Peduzzi P, Concato J, Kemper E, Holford TR, Feinstein AR. A simulation study of the number of events per variable in logistic regression analysis. J Clin Epidemiol 1996; 49: 1373-1379.

34 Zweig MH, Campbell G. Receiver-operating characteristic (ROC) plots: a fundamental evaluation tool in clinical medicine. Clin Chem 1993; 39: 561-577.

35 DeVivo MJ. Epidemiology of traumatic spinal cord injury: trends and future implications. Spinal Cord 2012, 50: 365-372.

36 New PW, Rawicki HB, Bailey MJ. Nontraumatic spinal cord injury rehabilitation: pressure ulcer patterns, prediction, and impact. Arch Phys Med Rehabil 2004; 85: 87-93.

37 Hammond M, Bozzacco V, Stiens S, Buhrer R, Lyman P. Pressure ulcer incidence on a spinal cord injury unit. Adv Wound Care 1994; 7: 57.

38 Taghipoor KD, Arejan RH, Rasouli MR, Saadat S, Moghadam M, Vaccaro AR et al. Factors associated with pressure ulcers in patients with complete or sensory-only preserved spinal cord injury: is there any difference between traumatic and nontraumatic causes? Clinical article. J Neurosurg 2009; 11: 438-444.

39 Scheel-Sailer A, Wyss A, Boldt C, Post M, Lay V. Prevalence, location, grade of pressure ulcers and association with specific patient characteristics in adult spinal cord injury patients during the hospital stay: a prospective cohort study. Spinal Cord 2013; 51: 828-833.

40 Fuhrer MJ, Garber SL, Rintala DH, Clearman R, Hart KA. Pressure ulcers in communityresident persons with spinal cord injury: prevalence and risk factors. Arch Phys Med Rehabil 1993; 74: 1172-1177.
41 Celani MG, Spizzichino L, Ricci S, Zampolini M, Franceschini M. Spinal cord injury in Italy: a multicenter retrospective study. Arch Phys Med Rehabil 2001; 82: 589-596.

42 Idowu O, Yinusa W, Gbadegesin S, Adebule G. Risk factors for pressure ulceration in a resource constrained spinal injury service. Spinal Cord 2011; 49: 643-647.

43 McKinley WO, Jackson AB, Cardenas DD, De Vivo MJ. Long-term medical complications after traumatic spinal cord injury: a regional model systems analysis. Arch Phys Med Rehabil 1999; 80: 1402-1410.

44 Krishnan S, Karg PE, Boninger ML, Brienza DM. Association between presence of pneumonia and pressure ulcer formation following traumatic spinal cord injury. J Spinal Cord Med 2016; 40: 415-422.

45 Krishnan S, Vodovotz Y, Karg PE, Constantine G, Sowa GA, Constantine FJ et al. Inflammatory mediators associated with pressure ulcer development in individuals with pneumonia after traumatic spinal cord injury: a pilot study. Arch Phys Med Rehabil (e-pub ahead of print 25 January 2017; doi: 10.1016/j.apmr.2016.12.018).

46 Esposito S, Noviello S, Leone S. Catheter-associated urinary tract infections: epidemiology and prevention]. Infez Med 2008; 16: 130.

47 Gould CV, Umscheid CA, Agarwal RK, Kuntz G, Pegues DA. Guideline for prevention of catheter-associated urinary tract infections 2009. Infect Control Hosp Epidemiol 2010; 31: 319-326.

48 Grey JE, Harding KG, Enoch S. Pressure ulcers. Br Med J 2006; 7539: 472.

49 Brandeis GH, Ooi WL, Hossain M, Morris JN, Lipsitz LA. A longitudinal study of risk factors associated with the formation of pressure ulcers in nursing homes. J Am Geriatr Soc 1994; 42: 388 